Fortnightly Review

\title{
Diagnosis and treatment of nasal polyps
}

\author{
Valerie J Lund
}

\section{poly-pous many-footed}

Nasal polyps have been a medically recognised condition since the time of the ancient Egyptians and their removal with a snare was described by Hippocrates, a method which persisted well into the second half of the 20th century.' Interestingly, only man and the chimpanzee are affected by this condition.

\section{Aetiology}

No single predisposing disease can be implicated in

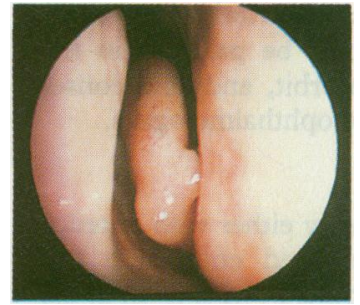

FIG 1-Endoscopic photograph showing localised area of polypoid change where mucosa of middle turbinate touches lateral wall in left nasal cavity

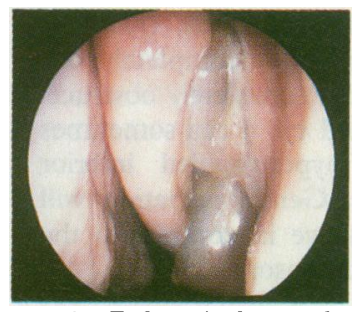

FIG 2-Endoscopic photograph showing more extensive polyposis in the right middle meatus between middle turbinate and lateral wall
University College London Medical School, Institute of Laryngology and Otology, London WC1X 8DA

Valerie J Lund, professor in rhinology

$B M \Im$ 1995;311:1411-4 the formation of polyps, though they may be associated with several other diseases (table), notably non-allergic (intrinsic) asthma and aspirin intolerance or sensitivity. No evidence exists, however, for an allergic

\begin{tabular}{lrr|} 
Percentage prevalence of nasal polyposis & \\
Normal population & 1 \\
Allergic patients & $1 \cdot 5$ \\
Asthmatic adults: & 7 \\
$\quad$ Non-allergic & 13 & \\
Aspirin intolerance & 36 & \\
Atopy & 5 & \\
Patients with “allergic" fungal sinusitis & 80 \\
Patients with cystic fibrosis & 18 \\
Patients with Kartagener's syndrome & 27 \\
& & \\
\end{tabular}

origin. ${ }^{2}$ In allergic rhinitis the prevalence of symptomatic nasal polyps is low $(1.5 \%)$, similar to that in the normal population (1\%). Since the advent of nasal endoscopy, however, any area of mucosal content may be associated with localised oedema or "polypoid" change, particularly in the middle meatus (fig 1 ) ${ }^{3}$ It is not known whether this change is the progenitor of gross polyposis (fig 2) and, if so, what factors determine progression of the disease process. Nasal polyps seem to be far more common than previous clinical studies have shown. Larsen et al reported that, with careful endoscopic examination of cadavers, a quarter of individuals had polyps originating in the sinus ostia or recesses of the lateral nasal wall without a history of sinonasal disease. ${ }^{4}$

Polyps do not seem to arise from all nasal and sinus mucosa, having a predilection for the middle turbinate, middle meatus, and ethmoids, while discrete polyps are rarely seen on the inferior turbinate or septum.

The role of infection is thought to be important by some in the genesis of polyp formation. This is based on experimental models in which multiple epithelial disruptions with proliferating granulation tissue have been initiated by bacterial infection with Streptococcus pneumoniae, Staphylococcus aureus, or Bacteroides

\section{Summary points}

- The aetiology of nasal polyposis is unknown though the condition is more common in certain medical disorders

- Nasal polyps must be distinguished from more serious pathology such as neoplasia

- Treatment is a combination of surgery and drugs; drug treatment is usually required long term

fragilis (all common pathogens in sinusitis) or Pseudomonas aeruginosa, which is often found in cystic fibrosis. ${ }^{56}$

The triad of nasal polyps, aspirin intolerance, and asthma was first described by Widal et al in $1922^{7}$ and represents the most aggressive form of the disease. Most often, the asthma precedes the clinical appearance of the polyps. The pathological mechanism of aspirin intolerance is through inhibition of the cyclooxygenase pathway of arachidonic metabolism resulting in an increased production of lipoxygenase metabolites, the leukotrienes $\mathrm{LTC}_{4}, \mathrm{LTD}_{4}$ and $\mathrm{LTE}_{4}$, which can cause acute bronchospasm, mucus hypersecretion, mucosal oedema, and increased concentrations of neutrophils and eosinophils in the exudate. Any of the non-steroidal anti-inflammatory drugs can cross react with aspirin to produce these symptoms in individuals who are intolerant of aspirin.

Nasal polyps seem to occur more often in men, and their prevalence increases in both sexes with age to reach a peak in those aged 50 years or older. In asthmatic people aged over 40 the prevalence is four times greater than in asthmatic people under $40(12 \cdot 4 \%$ $v 3.1 \%, \mathrm{P}<0.01) .{ }^{8}$ Any child of 16 years or younger who presents with nasal polyps must be regarded as having cystic fibrosis until proved otherwise. ${ }^{9}$ Doctors should be suspicious, however, of the presence of an encephalocele in any infant who has a unilateral nasal mass.

The association of cystic fibrosis with nasal polyposis was made by Lurie in $1957,{ }^{10}$ though the true prevalence is uncertain, with figures ranging from 3\% to $48 \%$. Cystic fibrosis is the predominant cause of polyps in children and may be the first presenting problem in some individuals.

\section{Histopathology}

Macroscopically polyps are pale bags of oedematous tissue arising most commonly in the clefts of the middle meatus and prolapsing into the nasal cavity. Their paleness is generally due to poor blood supply, 
but with time they may become fleshy and reddened due to squamous metaplasia. They are most often bilateral, and indeed any unilateral lesion should be regarded as suspicious of neoplasia.

The most common histological type is an oedematous eosinophilic polyp, characterised by goblet cell hyperplasia and thickening of the basement membrane, with a predominantly eosinophilic infiltrate. In all, $85-90 \%$ of nasal polyps are of this type. It is important that all polyp tissue removed surgically should be submitted for histological examination, and if medical treatment alone is used, some representative material should be examined to exclude the possibility of neoplasia. Both benign tumours (such as inverted papilloma) and malignancy (such as mucinous adenocarcinoma) can mimic or coexist with "ordinary" nasal polyps.

Occasionally large, single benign polyps arise in the maxillary antrum and prolapse into the nose, enlarging to fill the nasopharynx - antrochoanal polyps (figs 3 and 4). These are said to be more common in males and are of unknown aetiology. They probably arise from the floor or lateral wall of the sinus and prolapse through an accessory ostium into the nasal cavity. Imaging should always be performed to distinguish an antrochoanal polyp from a tumour such as an angiofibroma.

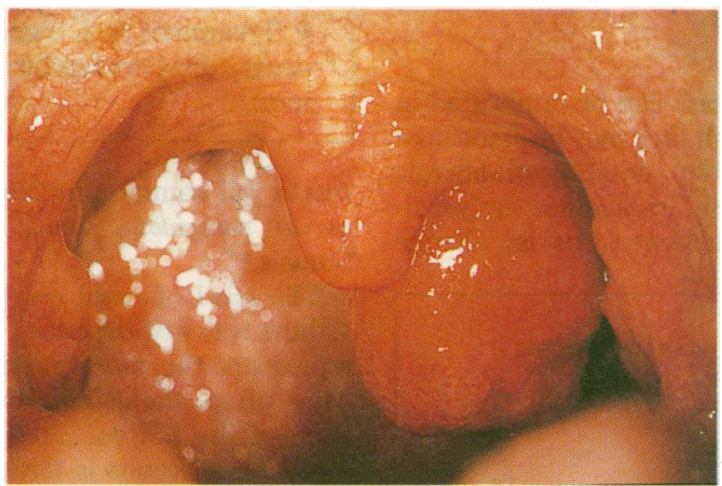

FIG 3-View of antrochoanal polyp presenting in oropharynx behind soft palate

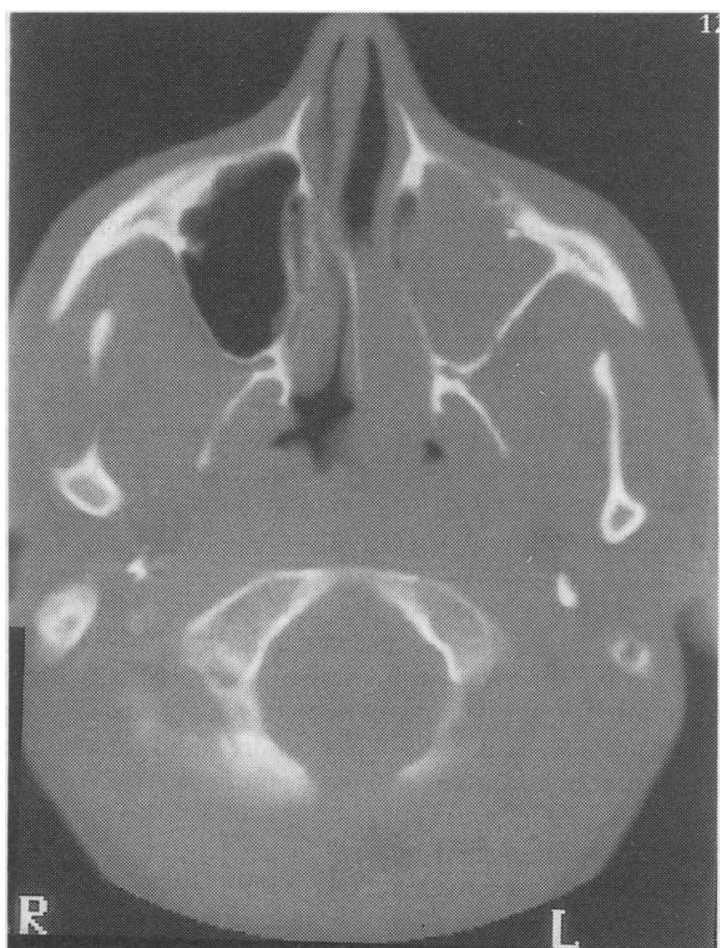

FIG 4-Axial computed tomogram showing opacification of left maxillary sinus due to antrochoanal polyp presenting in posterior nasal cavity and nasopharynx
If thick, inspissated, greeny brown secretion is seen in association with polyps then the possibility of a fungal infection should immediately be suspected, and it is often the histopathologist rather than the microbiologist who will confirm the diagnosis by specific staining for fungal hyphae as fungus is often difficult to culture.

\section{Diagnosis}

CLINICAL SYMPTOMS

The predominant complaint is nasal obstruction, which is constant but will vary in severity with the size of the polyps. The obstruction may affect the quality of the voice. Many patients also complain of watery anterior rhinorrhoea or mucopurulent postnasal drip, or both, but rarely of much facial pain or headaches despite ostensibly obstructed sinuses. Partial or complete loss of smell-plus a concomitant effect on taste, with loss of the subtleties of flavour-is a characteristic feature of nasal polyps.

When polyps begin in young adults or at an earlier age there will be a degree of ethmoidal expansion that may produce substantial hypertelorism. ${ }^{11}$ At least a third of patients also have lower respiratory tract symptoms, usually related to asthma. Occasionally, polyps may be associated with the development of a mucocele, usually in the frontoethmoidal sinus complex. ${ }^{12}$ This is an epithelial lined sac, completely filling the sinus and capable of bone erosion and expansion, which produces slow displacement of the globe laterally, inferiorly, and anteriorly with some degree of diplopia. A mass may be palpable in the superomedial quadrant of the orbit, and as a consequence patients often present to ophthalmologists.

\section{EXAMINATION}

Examination of the nasal cavity either with anterior rhinoscopy or ideally with a rigid endoscope will readily enable visualisation of nasal polyps. Even in the absence of specialised equipment, the nose may be adequately examined with an aural speculum. The use of decongestants and local anaesthesia may improve examination but are not necessary in the presence of gross polyps. Polyps may be crudely categorised into (a) those confined to the middle meatus, (b) those beyond the middle meatus but not completely obstructing the nose, and (c) those completely obstructing the airway. To the untrained eye it can sometimes be difficult to distinguish hypertrophied inferior turbinates from nasal polyps. Gentle palpation will show that the polyp is insensitive in contrast to the turbinate which has an excellent sensory supply.

\section{SPECIAL INVESTIGATIONS}

Several objective tests are available to quantify the restriction in nasal airway-notably nasal inspiratory peak flow, anterior rhinomanometry, and acoustic rhinometry-but have not yet entered routine clinical practice. ${ }^{13}$ An assessment of lower respiratory tract function should always be performed in a patient with nasal polyps as some patients have covert asthma.

Several well validated quantitative tests of smell are now commercially available - such as the University of Pennsylvania Smell Identification Test-which may be of value in assessing response to treatment.

\section{IMAGING}

Plain sinus $x$ ray films are of limited value in the diagnosis of nasal polyps, although they may confirm the generalised opacification of all the sinuses. If any surgical intervention is contemplated a computed tomogram is preferable and indeed mandatory if an endoscopic ethmoidectomy is to be performed. The scan will show the anatomy-including any alterations 
wrought by the disease or by previous surgery-as well as the extent of the condition. Various degrees of sinus opacification will be seen up to and including a complete "white out," associated with ethmoidal expansion, some degree of decalcification, and even loss of bony septa, adjacent lamina papyracea, and skull base (fig 5). Direct coronal cuts performed on wide window widths are optimal, and if disease extends into the posterior ethmoids and sphenoid, axial sections should also be performed to define the relation of the optic nerves and carotid arteries to the sinuses. Several protocols are available for these studies. ${ }^{14}$ Imaging is of particular relevance if an encephalocele, fungal infection, or tumour is suspected.

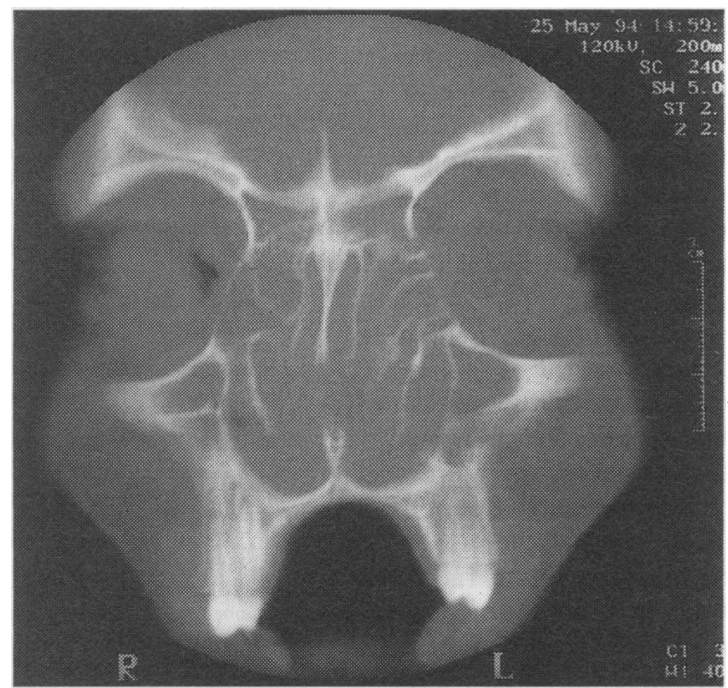

A chest $x$ ray examination may also be worth while in selected cases.

\section{Treatment}

Many people now believe that nasal polyposis represents a spectrum of disease in which both drugs and surgery usually play a part. As no single predisposing disease can account for the formation of polyps treatment must be directed towards the generalised inflammatory process. Treatment may be divided into primary treatment (medical or surgical) and secondary (or maintenance) treatment, which will be required long term, possibly for life. Treatment strategy should be determined on an individual basis. No single surgical approach has proved entirely curative, and patients often undergo repeated procedures during their life and receive long term drug treatment.

Once a polyp has been diagnosed, the patient is treated with a topical intranasal steroid preparation. The most effective is betamethasone sodium phosphate drops used in the "head down and forwards" position (fig 6). ${ }^{16}$ If a patient cannot adopt this position the drops should be administered by someone else, with the patient supine with the head hanging over the edge of the bed (fig 7). Administering the drops in the "head down and forwards" position can produce a dramatic shrinkage of the polyps within 48 hours. Once a pronounced reduction in size has been achieved, long term maintenance of the reduction may be provided by regular use of an intranasal steroid spray such as beclomethasone diproprionate, budesonide, or fluticasone propionate. ${ }^{17} 18$ Patients may be reassured about the long term safety of these drugs, in terms of both local and systemic effects.

A more dramatic way to treat polyps pharmacologically is to give oral steroids such as prednisolone or dexamethasone. A nine day course of dexamethasone

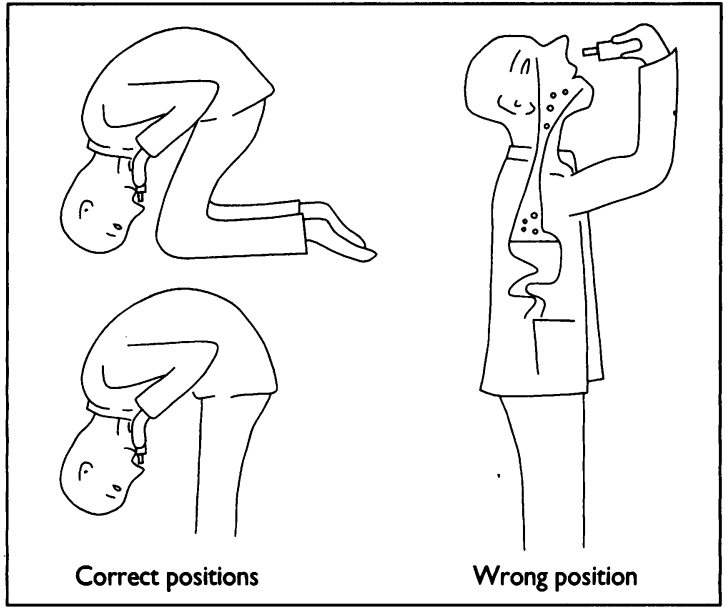

FIG 6-Diagram from patient information sheet showing "head down and forwards" positions for instilling nose drops

(12 $\mathrm{mg} / 3$ days, $8 \mathrm{mg} / 3$ days, $4 \mathrm{mg} / 3$ days) is usually successful in producing a dramatic reduction in the size and symptoms of nasal polyps. This regimen is contraindicated, however, in some individuals, including those with advanced osteoporosis, severe hypertension, diabetes mellitus, gastric ulceration, or herpetic keratitis.

The production of endogenous cortisol can be boosted with synthetic adrenocorticotrophic hormone-Synacthen Depot, two $1 \mathrm{mg}$ intramuscular injections given 48 hours apart (with the patient kept under observation for at least one hour after each injection). Corticotrophin and high dose oral steroids are most useful in a crisis when a rapid amelioration is needed, and such interventions may be given three to four time a year in most adults.

A study by Lildholt et al ${ }^{19}$ comparing nasal polypectomy with a single intramuscular injection of steroid in 53 patients, showed a similar benefit in both groups over the following year, during which both groups took a maintenance dose of intranasal beclomethasone. Not all patients, however, will be symptomatically controlled with drug treatment alone, and most need surgery at some point in their clinical course.

SURGERY

The surgical options may be broadly divided into intranasal or external approaches, or combinations of these. Intranasal surgery encompasses everything from simple snare polypectomy to radical ethmofrontosphenoidectomy with fenestration of the maxillary sinuses. Visualisation may be with head light, fibreoptically illuminated nasal speculum, microscope, or rigid endoscope. In modern rhinological practice, if more than a simple polypectomy is contemplated, the procedure should be preceded by computed tomography to show anatomic variation and distortion due

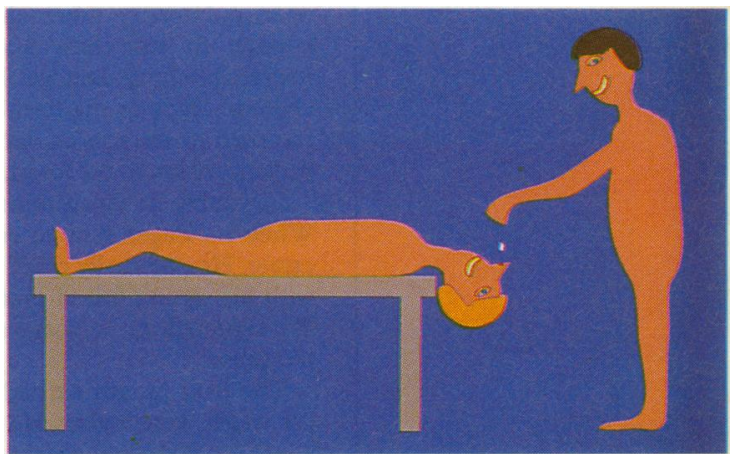

FIG 7-Alternative position for instilling nose drops for patients unable to adopt "head down and forwards" position 


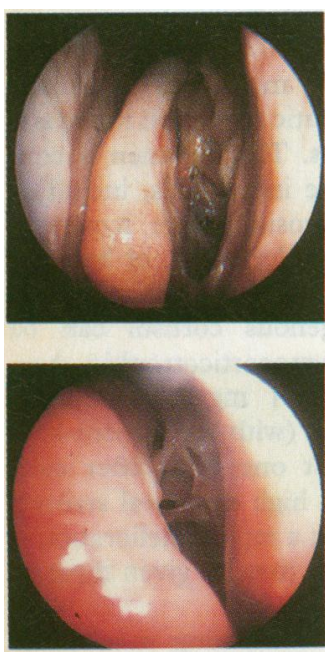

FIG 8-Top: Surgical cavity in patient shown in figure 2 after endoscopic polypectomy at two weeks. Bottom: Close up endoscopic view of same ethmoidal cavity at three month after endoscopic polypectomy with continued use of topical intranasal steroid to disease and the effects of any previous surgery.

In the past, if conventional snare polypectomy had been performed in one patient on increasingly frequent occasions, some surgeons advocated a more radical exenteration of the sinuses and nasal cavity via an external approach. This had the advantage of lengthening the symptom free interval compared with conventional polypectomy but rarely effected a cure. ${ }^{20}$ With the advent of endoscopic sinus surgery the same degree of surgical clearance is possible but with the advantage of avoiding facial scars. Such surgery, however, requires a high degree of expertise to achieve optimum results without complication. When endoscopic sinus surgery is undertaken the sinus system may be approached anteriorly via the middle meatus, with the amount of tissue removal tailored to the degree of pathology encountered. ${ }^{21}$ Alternatively, a "back to front" approach may be deemed more prudent when there has been previous extensive surgery. ${ }^{22}$ This involves identifying the sphenoid first and using the skull base and lateral wall as landmarks, progessively clearing the ethmoid sinuses from posterior to anterior. In both cases a surgical cavity is created into which drugs may be more advantageously instilled (fig 8).

Surgery may be performed under local or general anaesthesia. Advocates of local anaesthesia believe that it provides a better surgical field due to less bleeding and has the advantage of patient awareness if the orbit or intracranial fossa is breached, though this is certainly not an infallible method to avoid serious complications. With general anesthesia, the use of topical vasoconstricting agents combined with raising the head provides a generally excellent operating field. With both local and general anaesthesia some nasal bleeding usually occurs in the first few hours after surgery which is rarely severe but may necessitate an overnight stay in hospital. Postoperatively, patients are usually prescribed some form of nasal douche, and a topical intranasal steroid, together with a broad spectrum antibiotic if infected sinus secretions are found during surgery.

Thus in most cases patients' symptoms may be controlled by a combination of judicious surgery and long term topical intranasal steroid preparations (fig 9).

1 Wright J. History of laryngology and rhinology. St Louis: Lea and Febiger, 1893:57-9.

2 Drake-Lee AB, Lowe D, Swanston A, Grace A. Clinical profile and recurrence of nasal polyps. $₹$ Laryngol Otol 1984;98:783-93.

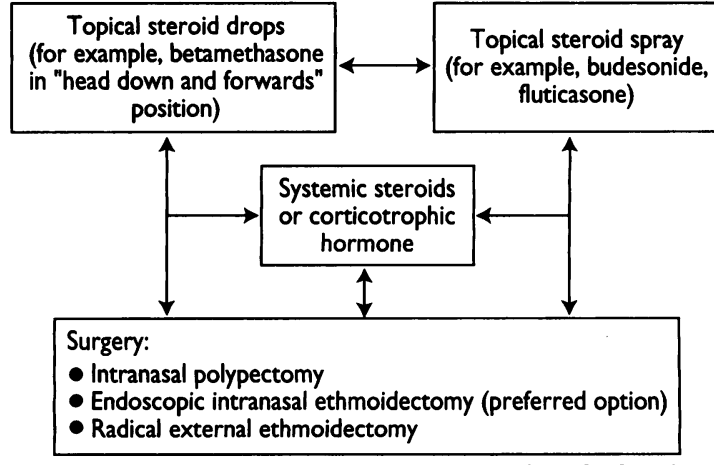

FIG 9-Algorithm of drug and surgical treatments of nasal polyposis

3 Stammberger H. Functional endoscopic sinus surgery. Philadelphia: Decker, 1991:217-32.

4 Larsen PL, Tos M, Baer S. En block removal of the ethmoid and ostiomeatal complex in cadavers, with a practical application. Rhinology 1964;32(2): $62-4$.

5 Norlander T, Fukami M, Wetrin KM, Stierna P, Carsloo B. Formation of mucosal polyps in the nasal and maxillary sinus cavities by infection. Otolaryngol Head Neck Surg 1993;109.522-9.

6 Norlander T, Westrin KM, Fukami M, Stierna P, Carlsőo B. Experimentally induced polyps in the sinus mucosa. Laryngoscope (in press).

7 Widal MF, Abrami P, Lermoyez J. Anaphylaxie et idiosyndraise. Presse Med 1922;30:189.

8 Settipane GA, Chafee FH. Nasal polyps in asthma and rhinitis: a review of 6,037 patients. F Allergy Clin Immunol 1977;59:17-21.

9 Schwachman H, Kulczychi IL, Mueller HL, Flake CG. Nasal polyposis in patients with cystic fibrosis. Pediatrics 1962;30:389-410.

10 Lurie H. Cystic fibrosis of the pancreas and the nasal mucosa. Ann Otol Rhinol Laryngol 1959;68:478.

11 Lund VJ, Lloyd GAS. Radiological changes associated with benign nasal polyps. F Laryngol Otol 1983;97:503-10.

12 Lund VJ. Anatomical considerations in the aetiology of fronto-ethmoidal mucocoeles. Rhinology 1987;25:83-8.

13 Lund VJ. Nasal obstruction-office evaluation. Otolaryngol Clin North Am 1992;25:803-16.

14 Zinreich SJ, Kennedy DW, Rosenbaum AE, Gayler BW, Kumar AJ, Stammberger $H$. Paranasal sinuses: CT imaging requirements for endoStammberger H. Paranasal sinuses: CT imaging

15 Lloyd GAS, Lund VI, Scadding GK. Computerised tomography in the preoperative evaluation of functional endoscopic sinus surgery. $f$ Laryngol Otol 1991;105:181-5.

16 Chalton R, Mackay IS. Double blind, placebo controlled trial of betamethasone nasal drops for nasal polyposis. $B M \mathcal{F} 1985 ; 291: 788$.

7 Ruhno J, Andersson BN, Denburg J, Andersson M, Hitch D, Lapp P, et al. A double-blind comparison of intranasal budesonide with placebo for nasal polyposis. F Allergy Clin Immunol 1990;86:946-53.

18 Mygind N, Brahe Pedersen C, Prytz S, Sorensen H. Treatment of nasal polyps with intranasal beclomethasone dipropionate aerosol. Clin Allerg 1975;5: 159-64.

19 Lildholdt T, Fogstrup J, Gammelgaard N, Kortholm B, Ulsoe C. Surgical versus medical treatment of nasal polyps. Acta Otolaryngol (Stockh) 1988; 105:140-3.

20 Tos M, Drake-Lee A, Lund VJ, Stammberger H. Treatment of nasal polypsmedication or surgery. Rhinology 1989;8(suppl):45-9.

21 Stammberger H, Posawetz W. Functional endoscopic sinus surgery: concept, indications and results of the Messerklinger technique. Eur Arch Otorhinoindications and results of

22 Wigand ME. Endoscopic surgery of the paranasal sinuses and anterior skull base. Stuttgart, New York: Thieme, 1990.

\section{A MEMORABLE PATIENT}

\section{Chemotherapy cannot combat poverty}

I first met this 20 year old man in 1992 in his isolated village in Tamil Nadu in India. He lived with his elderly, widowed father, who was deaf, in a crumbling stone and mud house and earned a hopelessly low income on the coffee estates. He had sought help for his symptoms from open pulmonary tuberculosis only when they were bad enough to stop him working. He already had some irreversible lung damage. He was given a short course of chemotherapy in the health centre and six weeks later he returned to work in an orange orchard, accessible only by walking $10 \mathrm{~km}$ in the forest.

Inevitably he failed to keep his follow up appointment. When messages sent to recall him failed we visited the estate. He eventually came to see us with a broad smile and with his sarong full of oranges for us as a peace offering. We repeated the message about the need to continue the treatment.

The next month he had left for another district where the wages were higher. He returned five months later with a relapse of the symptoms. I pleaded with him to stay free at our base hospital for at least three months. He said that he could not stay in peace in hospital while his father was hungry. I saw him the next month on top of a guava tree picking fruit; on request he collected his next month's drugs which were given under supervision by a neighbour.

Five months later when I saw him again in his village he was critically ill and in shock. Treatment would mean transport by horseback, then bus, to the base hospital with no guarantee of survival and the likelihood of drug resistant tuberculosis. I decided not to treat him and he died the next day.

Tuberculosis programmes, however efficient, can never be enough in themselves for those in extreme poverty. Only those who have visited the homes of such patients and participated in their struggles can understand why. The solution to tuberculosis in poor countries lies, as it did in the West in the nineteenth century, in the need for a solution to poverty.-RAJKUMAR RAMASAMY is a consultant physician in Oddanchatram, India 